\title{
Why Some Interfaces can Never be Sharp
}

\author{
D. A. Muller*, N. Nakagawa**, H. Y. Hwang** \\ *School of Applied and Engineering Physics, Cornell University, Ithaca, NY 14853 \\ **Department of Advanced Materials, University of Tokyo, Kashiwa, Chiba 277-8561, Japan
}

A central goal in producing device-quality oxide electronics and ferroelectrics has been the growth of atomically-abrupt heterointerfaces [1]. What has not been generally recognized however is that the goals of producing electrically and atomically abrupt interfaces between polar and nonpolar layers are mutually incompatible. Fig. 1 details how the distribution of charges across the interface plane can make the difference between an electrostatic potential that diverges with thickness (for ideal termination between neutral and polar layered materials) and one that simple produces a small, but finite band offset (by adding an interface dipole). This is the bulk analogy of the surface reconstructions found in polar systems where a material terminated along a bulk polar plane has a net charge and a divergent surface energy. The consequence for growing polar materials on nonpolar substrates (like GaAs on $\mathrm{Si}$ or $\mathrm{Ge}$ ) is a catastrophic roughening during growth, unless the composition is graded at the interface to ensure there is no net formal charge [2]. This results in a microscopically rough interface, and in many cases also a measurable electrical band offset [2]. Unlike conventional semiconductors, for multivalent oxides, physical roughness is not the only option - atomically abrupt interfaces are allowed if electrons can be redistributed at lower energy cost than redistributing ions. In Fig $1 \mathrm{~b}$ by constructing each layer from neutral atoms then transferring $1 / 2 \mathrm{e}^{-}$to neighboring layers to obtain the bulk ionic structure leaves the overall structure neutral. At the interface, the Ti ion picks up an extra $1 / 2$ electron, becoming $\mathrm{Ti}^{3.5+}$. The extra half electron at the Ti-La terminated interface should be physically detectable by EELS.

The choices of materials is not random. $\mathrm{SrTiO}_{3}$ is the workhorse oxide semiconductor and $\mathrm{LaAlO}_{3}$ is a closely lattice-matched insulator, useful as a gate dielectric for field-effect devices. Recent work on $\mathrm{SrTiO}_{3} / \mathrm{LaAlO}_{3}$ [001] interfaces has found that Ti-La interfaces are conducting, but $\mathrm{Sr}-\mathrm{Al}$ interfaces are insulating [3]. Fig. 2 shows a highly condensed summary of EELS line profiles across $\mathrm{SrTiO}_{3} / \mathrm{LaAlO}_{3}$ interfaces. The Ti $\mathrm{L}$ and $\mathrm{O}-\mathrm{K}$ edges are fitted to reference spectra of $\mathrm{Ti}^{3+} / \mathrm{Ti}^{4+}$ and $\mathrm{O}-\mathrm{K}$ in $\mathrm{SrTiO}_{3}, \mathrm{LaTiO}_{3}$ and oxygen deficient $(\delta=0.25) \mathrm{SrTiO}_{2.75}$ [4,5]. The quality of the fit can be evaluated from examining variations in the residual. We find that for the Ti-La terminated interface, excess electrons are found on the Ti sites (non-zero $\mathrm{Ti}^{3+}$ ), but not for the $\mathrm{Sr}-\mathrm{Al}$ termination (where an extra $1 / 2$ hole would be expected theoretically). Instead significant compensating oxygen vacancies are present and no free holes are found, probably explaining the electrical asymmetry. Controlling the interface termination lets us tune between insulator and conductor, trading chemical for electronic roughness [6].

References

[1] C.H. Ahn, J.-M. Triscone, and J. Mannhart, Nature, 2003. 4241015.

[2] W. A. Harrison et al., Phys. Rev. B18, (1978) 4402.

[3] A. Ohtomo, H.Y. Hwang, Nature, 427 (2004) 423.

[4] D. A. Muller et al., Nature, 430 (2004) 657.

[5] A. Ohtomo, D.A. Muller, J.L. Grazul, and H.Y. Hwang, Nature, 419 (2002) 378

[6] This work was supported by the Cornell Center for Materials Research (CCMR), a Materials Research Science and Engineering Center of the NSF (DMR-0079992). 


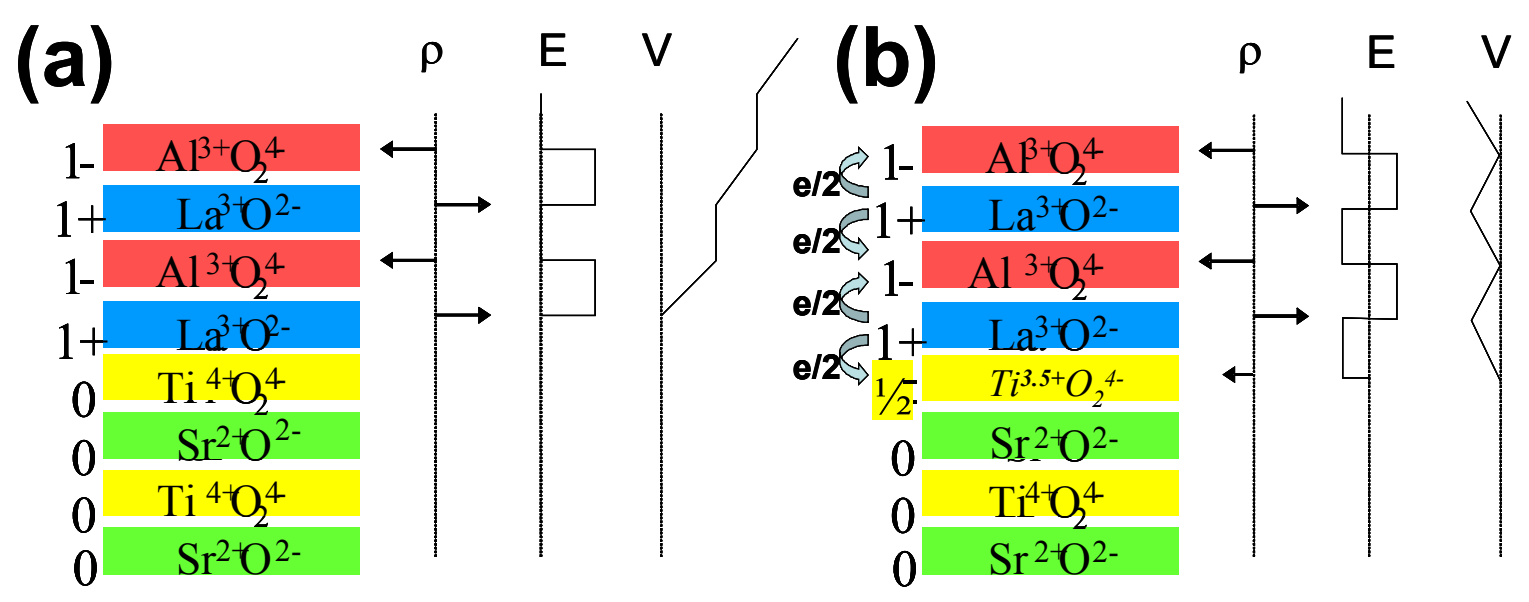

FIG. 1. The polar catastrophe illustrated for La-terminated [001] $\mathrm{LaAlO}_{3}$ on $\mathrm{SrTiO}_{3}$ (a) The unreconstructed interface has neutral (001) planes in $\mathrm{SrTiO}_{3}$, but the (001) planes in $\mathrm{LaAlO}_{3}$ have alternating net charges $(\rho)$. This produces a non-negative electric field $(E)$, leading in turn to an electric potential (V) that diverges with thickness. (b) The divergence catastrophe can be avoided if $1 / 2$ an electron is added to last Ti layer. This produced an interface dipole that causes the electric field to oscillate about 0 and the potential remains finite (see text for discussion of the arrows).

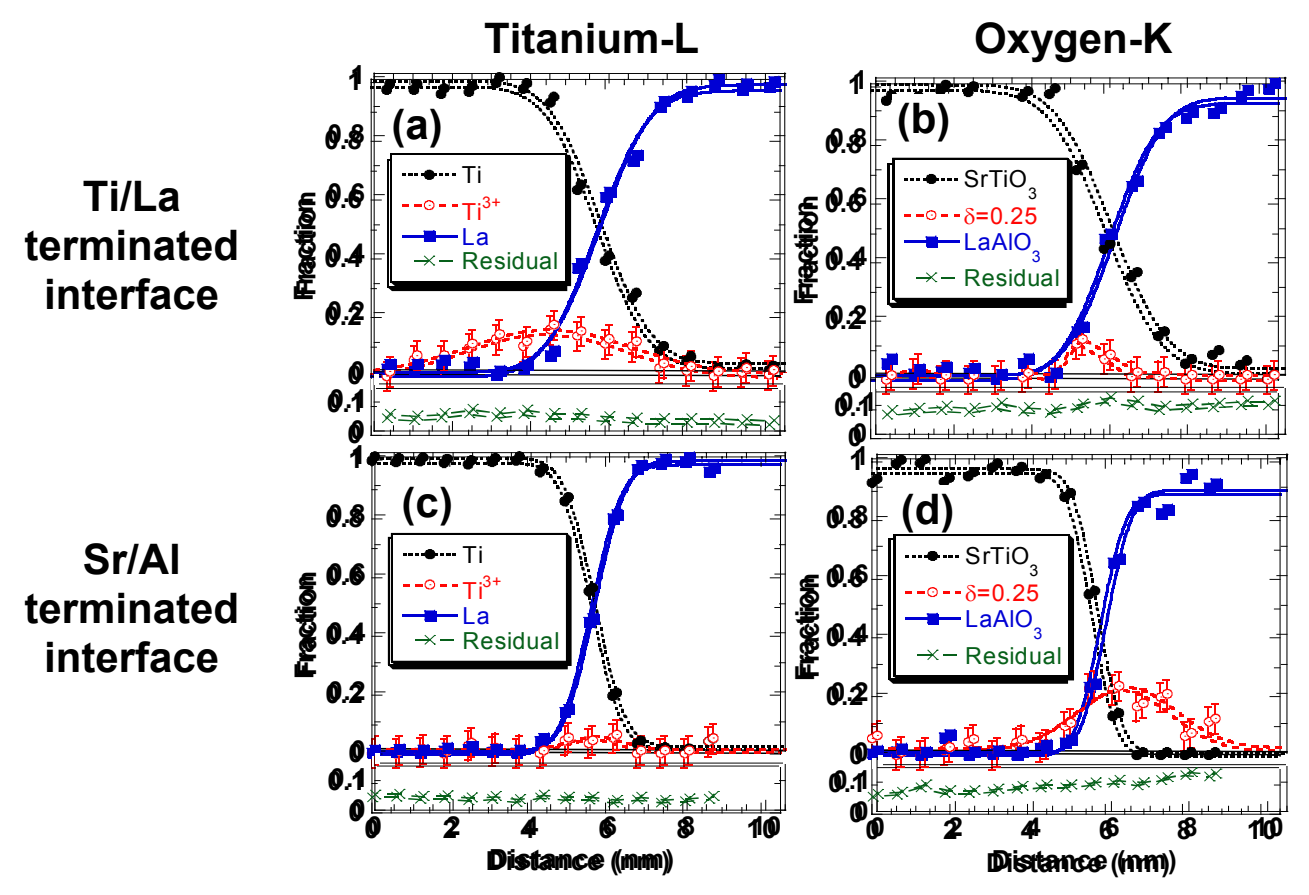

FIG. 2. Physical charge profiles across $\mathrm{LaAlO}_{3} / \mathrm{SrTiO}_{3}$ interfaces from EELS. Excess electrons can be detected from the $\mathrm{Ti}^{3+}$ EELS fingerprint $(\mathrm{a}, \mathrm{c})$. Instead of excess holes, the system compensates by producing oxygen vacancies $\left(\delta=0.25\right.$ is the $\mathrm{O}-\mathrm{K}$ spectrum from oxygen deficient $\left.\mathrm{SrTiO}_{3-\delta}\right)-$ panels b,c. The Ti/La terminated interface which formally has a net excess $0.5 \mathrm{e}^{-}$, excess $\mathrm{Ti}^{3+}$ is as expected detected (a). The Sr/Al terminated interface formally should have a net 0.5 extra hole, but is instead insulating, has no excess $\mathrm{Ti}^{3+}$, and instead shows a large excess of oxygen vacancies $(\mathrm{d})$. 